\title{
A small contribution to mitigate the collision of transmissible and chronic diseases, exemplified by the management of hypertension during the COVID-19 pandemic
}

\author{
Gloria Valdés $\mathbb{1}^{1} \cdot$ Carlos Zúñiga ${ }^{2,3}$
}

Received: 15 July 2020 / Revised: 8 November 2020 / Accepted: 25 November 2020 / Published online: 10 December 2020

(c) Crown 2020

We want to take up the challenge posed by Nadar and cols. in their May editorial about managing hypertension during the COVID-19 pandemic [1]. Their concern that patients with chronic illnesses would be forgotten in the fight against the paradigm of a transmissible virus and result in collateral damage reached the public domain since June [2]. In mid October the number of new confirmed cases is still increasing in the Americas, South-East Asia and Europe according to the World Health Organization [3]; lockdowns have been reinstalled in various zones and widely available vaccines are far from around the corner. In the present situation it is urgent to mitigate the collision of nontransmissible conditions with the rapid spread of the novel COVID infection by new patterns of interaction between all the protagonists involved in health care.

An unexpected and beneficial collateral effect of the prolonged worldwide sanitary crisis is the shift in the balance between critical and stable health conditions by addressing most of the control of hypertension and other chronic conditions to the virtual attention. The American Society of Preventive Cardiology has proposed virtual team care in order to override the pandemic [4]. This relatively new mode has been well accepted by patients and validated by randomized clinical trials, metanalysis and systematic reviews that show similar blood pressure control than the conventional form, but if accompanied by education and

Gloria Valdés

gvaldesst@gmail.com

1 Nephrology Department, Facultad de Medicina, Pontificia Universidad Católica, Santiago, Chile

2 Facultad de Medicina, Universidad Católica de la Santísima Concepción, Concepción, Chile

3 Coordinator, Telenephrology Unit, Hospital Higueras, Talcahuano, Chile counselling obtains extra benefits [5-8]. In addition, telemedicine offers several advantages (Table 1), including more equal and patient-centered health care in times in which vulnerable groups increase, receive the greatest economic and sanitary toll and require individual support. The way to virtual attention has been paved by affordable and validated automatic blood pressure monitors that permit adequate home controls when performed under the recommendations of the American Heart and the American Medical Associations [9]. Lastly, by the massive incorporation of virtual connections to daily life derived by the restrictions imposed by COVID-19.

Free access to ongoing validated protocols would help to rapidly integrate this relatively new form of care, but in the meantime we propose a simple and inexpensive framework that might be adapted to local practices with a pen, paper and digital connections (Fig. 1). This strategy is possible by incorporating graduates in training in the health careers to build data bases to contact patients while the risks of acquiring COVID-19 persist. Reaching out for patients potentiates the attachment between the health team and subjects under their care, which will improve adhesion to controls, therapy and recommendations. In this occasion it is necessary to identify whether the patient has a selfmeasurement device. In case he has none, pharmacies can play a role. In low income areas medical societies, pharmaceutical industries and profit and non-profit organizations among others must unite efforts to provide individual monitors.

Once the crisis is over, the continuity in the use of telehealth in hypertension will require returning to previous protocols, guidelines and quality standards that guarantee safety, ethics, reliability and legal issues. But while health systems continue to be overloaded for an unpredictable time this - or other simple proposals - could contribute to return to a continuous combination of face-to-face and virtual attention that previously diagnosed hypertensives need. 
Table 1 Advantages of telemedicine in the care of hypertensives in times of COVID-19.

\section{Inherent to telemedicine}

- Virtual and telephonic visits for management of "uncomplicated" cases are well accepted by patients, who avoid transportation costs and time in waiting rooms.

- Primary care providers estimate televisits improve patient interaction and show rates compared to clinic/office visits reducing non show rates.

- Providers and administrators report a greater efficiency by triaging patients to different levels of care givers, which is especially important in public health services.

- Homogenizes the quality of health care.

- Facilitates a timely access to individuals in distant locations.

- Permits the incorporation of coordinated interventions in public health networks.

- Selects and expedites references to the scarce resource of specialists.

Under the COVID-19 pandemia

- Benefits hypertensive patients, a high risk group in which isolation is recommended.

- Reduces the stress of exposing himself and his family to viral transmition an important benefit in the face of the reactivity displayed by hypertensives.

- Facilitates the access to the health team, medications and controls of comorbidities.

- Involves the immediate family in the adhesion to pharmacological and nutritional personalized management.

- Permits the continuity of usual care under confinement and social distancing.

- Reduces community and hospital viral dissemination.

- Limits conventional controls to the need of a physical exam and emergencies.

Postpandemia

- Promotes the creation of telehealth programs to face COVID-19 recurring waves, or a steady state of low transmission, and to manage the demand of non-COVID-19 patients.

- Facilitates the transition from the "emergency model" to a sustainable state among health workers and patients, after reevaluating ethical, legal and financial aspects.

- Poses the challenge of incorporating ehealth to the paradigm of presential health care.

- Promotes creation of protocols, guidelines and quality standards for hypertension ecare.

- Permits continuing patient education through websites and social networks to stimulate self-care and responsibility regarding healthy life styles changes.

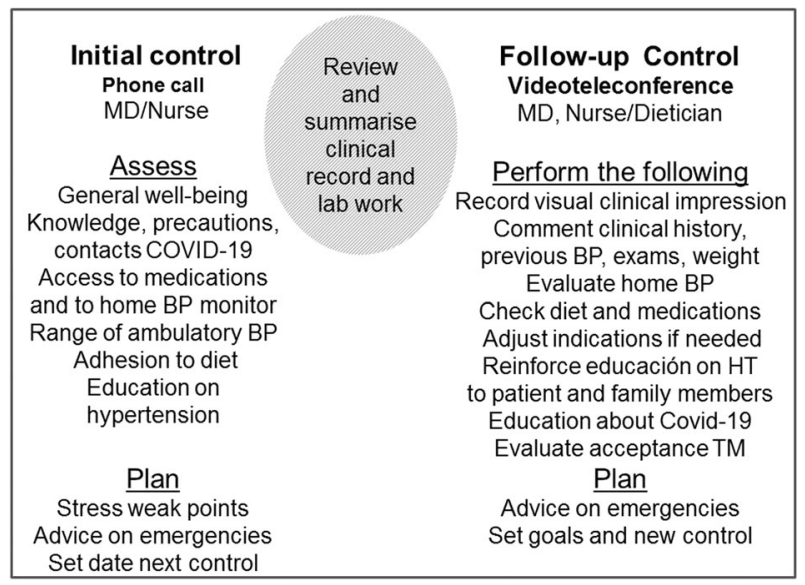

Fig. 1 Actions included in the first two controls with hypertensive patients (left and right pannel) separated by the review of the clinical file by the health team in the interim period (hatched circle).

\section{Compliance with ethical standards}

Conflict of interest The authors declare that they have no conflict of interest.

Publisher's note Springer Nature remains neutral with regard to jurisdictional claims in published maps and institutional affiliations.

\section{References}

1. Nadar SK, Tayebjee MH, Stowasser M, Byrd JB. Managing hypertension during the COVID-19 pandemic. J Hum Hypertens. 2020;34:415-7.

2. Mihaljevic T, Farrugia G How many more will die from fear of the coronavirus? https://www.nytimes.com/2020/06/09/opinion/corona virus-hospitals-deaths.html.

3. WHO Coronavirus Disease (COVID-19) Dashboard. https:// covid19. who.int/?gclid=CjwKCAjww5r8BRB6Eiw ArcckC0TJOpBxrIspInfGsNzrUGH71_3cmpMzL33RpxEz-_DO0M-PuMp-hoC-BwQAvD_BwE (accessed October 14 2020).

4. Khera A, Baum SJ, Gluckman TJ, Gulati M, Martin SS, Michos $\mathrm{ED}$, et al. Continuity of care and outpatient management for patients with and at high risk for cardiovascular disease during the COVID-10 pandemic: A scientific statement from the American Society for Preventive Cardiology. Am J Preventive Cardiol. 2020;1:100009.

5. Margolis KL, Asche SE, Dehmer SP, Bergdall AR, Green BB, Sperl-Hillen JM, et al. Long-term outcomes of the effects of home blood pressures telemonitoring and pharmacist management on blood pressure among adults with uncontrolled hypertension. Follow-up of a cluster randomized clinical trial. JAMA Netw Open. 2018;1:e181617.

6. Omboni S. Connected health in hypertension management. Front Cardiovasc Med. 2019;6:76.

7. Duan Y, Xie Z, Dong F, Wu Z, Lin Z, Sun N, et al. Effectiveness of home blood pressure telemonitoring: a systematic review and metaanalysis of randomised controlled studies. J Hum Hypertens. 2017;31:427-37.

8. Pellegrini D, Torlasco C, Ochoa JE, Parati G. Contribution of telemedicine and information technology to hypertension control. Hypertens Res. 2020;43:621-8.

9. Shimbo D, Artininan NT, Basile JN, Krakoff LR, Margolis KL, Rakotz MK, et al. Self-measured blood pressure monitoring at home: a Joint Policy Statement from the American Heart Association and American Medical Association. Circulation. 2020;142: e42-e63. 